\title{
Riemann-Liouville and Higher Dimensional Hardy Operators for NonNegative Decreasing Function in $L^{p(\cdot)}$ Spaces
}

\author{
Muhammad Sarwar, ${ }^{1}$ Ghulam Murtaza, ${ }^{2}$ and Irshaad Ahmed ${ }^{2}$ \\ ${ }^{1}$ Department of Mathematics, University of Malakand, Chakdara, Lower Dir 18800, Pakistan \\ ${ }^{2}$ Department of Mathematics, GC University, Faisalabad, Faisalabad 38000, Pakistan
}

Correspondence should be addressed to Muhammad Sarwar; sarwarswati@gmail.com

Received 23 May 2014; Accepted 6 August 2014; Published 26 August 2014

Academic Editor: Norio Yoshida

Copyright ( 2014 Muhammad Sarwar et al. This is an open access article distributed under the Creative Commons Attribution License, which permits unrestricted use, distribution, and reproduction in any medium, provided the original work is properly cited.

One-weight inequalities with general weights for Riemann-Liouville transform and $n$-dimensional fractional integral operator in variable exponent Lebesgue spaces defined on $\mathbb{R}^{n}$ are investigated. In particular, we derive necessary and sufficient conditions governing one-weight inequalities for these operators on the cone of nonnegative decreasing functions in $L^{p(x)} \operatorname{spaces}$.

\section{Introduction}

We derive necessary and sufficient conditions governing the one-weight inequality for the Riemann-Liouville operator

$$
R_{\alpha} f(x)=\frac{1}{x^{\alpha}} \int_{0}^{x} \frac{f(t)}{(x-t)^{1-\alpha}} d t \quad 0<\alpha<1
$$

and $n$-dimensional fractional integral operator

$$
I_{\alpha} g(x)=\frac{1}{|x|^{\alpha}} \int_{|y|<|x|} \frac{g(t)}{|x-t|^{n-\alpha}} d t \quad 0<\alpha<n,
$$

on the cone of nonnegative decreasing function in $L^{p(x)}$ spaces.

In the last two decades a considerable interest of researchers was attracted to the investigation of the mapping properties of integral operators in so-called Nakano spaces $L^{p(\cdot)}$ (see, e.g., the monographs $[1,2]$ and references therein). Mathematical problems related to these spaces arise in applications to mechanics of the continuum medium. For example, Ružicka [3] studied the problems in the so-called rheological and electrorheological fluids, which lead to spaces with variable exponent.

Weighted estimates for the Hardy transform

$$
\left(H_{1} f\right)(x)=\int_{0}^{x} f(t) d t, \quad x>0,
$$

in $L^{p(\cdot)}$ spaces were derived in the papers [4] for powertype weights and in [5-9] for general weights. The Hardy inequality for nonnegative decreasing functions was studied in $[10,11]$. Furthermore Hardy type inequality was studied in $[12,13]$ by Rafeiro and Samko in Lebesgue spaces with variable exponent.

Weighted problems for the Riemann-Liouville transform in $L^{p(x)}$ spaces were explored in the papers $[5,14-16]$ (see also the monograph [17]).

Historically, one and two weight Hardy inequalities on the cone of nonnegative decreasing functions defined on $\mathbb{R}_{+}$in the classical Lebesgue spaces were characterized by Arino and Muckenhoupt [18] and Sawyer [19], respectively.

It should be emphasized that the operator $I_{\alpha} f(x)$ is the weighted truncated potential. The trace inequity for this operator in the classical Lebesgue spaces was established by Sawyer [20] (see also the monograph [21], Ch.6 for related topics).

In general, the modular inequality

$$
\int_{0}^{1}\left|\int_{0}^{x} f(t) d t\right|^{q(x)} v(x) d x \leq c \int_{0}^{1}|f(t)|^{p(t)} w(t) d t
$$

for the Hardy operator is not valid (see [22], Corollary 2.3, for details). Namely, the following fact holds: if there exists a positive constant $c$ such that inequality $(*)$ is true for all 
$f \geq 0$, where $q ; p ; w$; and $v$ are nonnegative measurable functions, then there exists $b \in[0,1]$ such that $w(t)>0$ for almost every $t<b ; v(x)=0$ for almost every $x>b$, and $p(t)$ and $q(x)$ take the same constant values a.e. for $t \in(0 ; b)$ and $x \in(0 ; b) \cap\{v \neq 0\}$.

To get the main result we use the following pointwise inequalities:

$$
\begin{aligned}
& c_{1}(T f)(x) \leq\left(R_{\alpha} f\right)(x) \leq c_{2}(T f)(x), \\
& c_{3}(H g)(x) \leq\left(I_{\alpha} g\right)(x) \leq c_{4}(H g)(x),
\end{aligned}
$$

for nonnegative decreasing functions, where $c_{1}, c_{2}, c_{3}$, and $c_{4}$ are constants and are independent of $f, g$, and $x$, and

$$
\begin{gathered}
\operatorname{Tf}(x)=\frac{1}{x} \int_{0}^{x} f(t) d t, \\
H g(x)=\frac{1}{|x|^{n}} \int_{|y|<|x|} g(y) d y .
\end{gathered}
$$

In the sequel by the symbol $T f \approx T g$ we mean that there are positive constants $c_{1}$ and $c_{2}$ such that $c_{1} T f(x) \leq T g(x) \leq$ $c_{2} T f(x)$. Constants in inequalities will be mainly denoted by $c$ or $C$; the symbol $\mathbb{R}_{+}$means the interval $(0,+\infty)$.

\section{Preliminaries}

We say that a radial function $f: \mathbb{R}^{n} \rightarrow \mathbb{R}_{+}$is decreasing if there is a decreasing function $g: \mathbb{R}_{+} \rightarrow \mathbb{R}_{+}$such that $g(|x|)=f(x), x \in \mathbb{R}^{n}$. We will denote $g$ again by $f$. Let $p$ : $\mathbb{R}^{n} \rightarrow \mathbb{R}^{n}$ be a measurable function, satisfying the conditions $p^{-}=\operatorname{essinf}_{x \in \mathbb{R}^{n}} p(x)>0, p^{+}=\operatorname{esssup}_{x \in \mathbb{R}^{n}} p(x)<\infty$.

Given $p: \mathbb{R}^{n} \rightarrow \mathbb{R}_{+}$such that $0<p^{-} \leq p^{+}<\infty$ and a nonnegative measurable function (weight) $u$ in $\mathbb{R}^{n}$, let us define the following local oscillation of $p$ :

$$
\varphi_{p(\cdot), u}(\delta)=\underset{x \in B(0, \delta) \cap \operatorname{supp} u}{\operatorname{esssup}} p(x)-\underset{x \in B(0, \delta) \text { กsupp } u}{\operatorname{essinf}} p(x),
$$

where $B(0, \delta)$ is the ball with center 0 and radius $\delta$.

We observe that $\varphi_{p(\cdot), u}(\delta)$ is nondecreasing and positive function such that

$$
\lim _{\delta \rightarrow \infty} \varphi_{p(\cdot), u}(\delta)=p_{u}^{+}-p_{u}^{-},
$$

where $p_{u}^{+}$and $p_{u}^{-}$denote the essential infimum and supremum of $p$ on the support of $u$, respectively.

By the similar manner (see [10]) the function $\psi_{p(\cdot), u}(\eta)$ is defined for an exponent $p: \mathbb{R}_{+} \mapsto \mathbb{R}_{+}$and weight $v$ on $\mathbb{R}_{+}$:

$$
\psi_{p(\cdot), v}(\eta)=\operatorname{esssup}_{x \in(0, \eta) \cap \operatorname{supp} v} p(x)-\underset{x \in(0, \eta) \cap \operatorname{supp} v}{\operatorname{essinf}} p(x) .
$$

Let $D\left(\mathbb{R}_{+}\right)$be the class of nonnegative decreasing functions on $\mathbb{R}_{+}$and let $D R\left(\mathbb{R}^{n}\right)$ be the class of all nonnegative radially decreasing functions on $\mathbb{R}^{n}$. Suppose that $u$ is measurable a.e. positive function (weight) on $\mathbb{R}^{n}$. We denote by $L^{p(x)}\left(u, \mathbb{R}^{n}\right)$ the class of all nonnegative functions on $\mathbb{R}^{n}$ for which

$$
S_{p}(f)=\int_{\mathbb{R}^{n}}|f(x)|^{p(x)} u(x) d \mu(x)<\infty .
$$

For essential properties of $L^{p(x)}$ spaces we refer to the papers $[23,24]$ and the monographs $[1,2]$.

Under the symbol $L_{\mathrm{dec}}^{p(\mathrm{x})}\left(u, \mathbb{R}_{+}\right)$we mean the class of nonnegative decreasing functions on $\mathbb{R}_{+}$from $L^{p(x)}\left(u, \mathbb{R}^{n}\right) \cap$ $\operatorname{DR}\left(\mathbb{R}^{n}\right)$.

Now we list the well-known results regarding one-weight inequality for the operator $T$. For the following statement we refer to [18].

Theorem A. Let $r$ be constant such that $0<r<\infty$. Then the inequity

$$
\begin{array}{r}
\int_{0}^{\infty} v(x)(T f(x))^{r} d x \leq c \int_{0}^{\infty} v(x)(f(x))^{r} d x, \\
f \in L^{r}\left(v, \mathbb{R}_{+}\right), f \downarrow
\end{array}
$$

for a weight $v$ holds, if and only if there exists a positive constant $C$ such that for all $s>0$

$$
\int_{s}^{\infty}\left(\frac{s}{x}\right)^{r} v(x) d x \leq C \int_{0}^{s} v(x) d x .
$$
in $[18]$.

Condition (11) is called $B_{r}$ condition and was introduced

Theorem B (see [10]). Let $v$ be a weight on $(0, \infty)$ and $p$ : $\mathbb{R}_{+} \rightarrow \mathbb{R}_{+}$such that $0<p^{-} \leq p^{+}<\infty$, and assume that $\psi_{p(\cdot), v\left(0^{+}\right)}=0$. The following facts are equivalent:

(a) there exists a positive constant $c$ such that, for any $f \in$ $D\left(\mathbb{R}_{+}\right)$,

$$
\int_{0}^{\infty}(T f(x))^{p(x)} v(x) d x \leq C \int_{0}^{\infty}(f(x))^{p(x)} v(x) d x ;
$$

(b) for any $r, s>0$,

$$
\int_{r}^{\infty}\left(\frac{r}{s x}\right)^{p(x)} v(x) d x \leq C \int_{0}^{r} \frac{v(x)}{s^{p(x)}} d x ;
$$

(c) $p_{\left.\right|_{\text {supp } v}} \equiv p_{0}$ a.e. and $v \in B_{p_{0}}$.

Proposition 1. For the operators $T, H, R_{\alpha}$, and $I_{\alpha}$, the following relations hold:

(a)

$$
R_{\alpha} f \approx T f, \quad 0<\alpha<1, f \in D\left(\mathbb{R}_{+}\right)
$$

(b)

$$
I_{\alpha} g \approx H g, \quad 0<\alpha<n, g \in D R\left(\mathbb{R}^{n}\right) .
$$

Proof. (a) Upper estimate: represent $R_{\alpha} f$ as follows:

$$
\begin{aligned}
R_{\alpha} f(x)= & \frac{1}{x^{\alpha}} \int_{0}^{x / 2} \frac{f(t)}{(x-t)^{1-\alpha}} d t \\
& +\frac{1}{x^{\alpha}} \int_{x / 2}^{x} \frac{f(t)}{(x-t)^{1-\alpha}} d t \\
= & S_{1}(x)+S_{2}(x) .
\end{aligned}
$$


Observe that if $t<x / 2$, then $x / 2<x-t$. Hence

$$
S_{1}(x) \leq c \frac{1}{x} \int_{0}^{x / 2} f(t) d t \leq \operatorname{cTf}(x),
$$

where the positive constant $c$ does not depend on $f$ and $x$. Using the fact that $f$ is decreasing we find that

$$
S_{2}(x) \leq c f\left(\frac{x}{2}\right) \leq c T f(x) .
$$

Lower estimate follows immediately by using the fact that $f$ is nonnegative and the obvious estimate $x-t \leq x$ and $0<$ $t<x$. follows:

(b) Upper estimate: let us represent the operator $I_{\alpha}$ as

$$
\begin{aligned}
I_{\alpha} g(x)= & \frac{1}{|x|^{\alpha}} \int_{|y|<|x| / 2} \frac{g(y)}{|x-y|^{n-\alpha}} d y \\
& +\frac{1}{|x|^{\alpha}} \int_{|x| / 2<|y|<|x|} \frac{g(y)}{|x-y|^{n-\alpha}} d y \\
= & : S_{1}^{\prime}(x)+S_{2}^{\prime}(x) .
\end{aligned}
$$

Since $|x| / 2 \leq|x-y|$ for $|y|<|x| / 2$ we have that

$$
S_{1}^{\prime}(x) \leq \frac{c}{|x|^{n}} \int_{|y|<|x| / 2} g(y) d y \leq c H g(x) .
$$

Taking into account the fact that $f$ is radially decreasing on $\mathbb{R}^{n}$ we find that there is a decreasing function $f: \mathbb{R}_{+} \rightarrow \mathbb{R}_{+}$ such that

$$
S_{2}^{\prime}(x) \leq f\left(\frac{|x|}{2}\right) \cdot \frac{1}{|x|^{\alpha}} \int_{|x| / 2<|y|<|x|}|x-y|^{\alpha-n} d y .
$$

Let $F_{x}=\{y:|x| / 2<|y|<|x|\}$. Then we have

$$
\begin{aligned}
& \int_{F_{x}}|x-y|^{\alpha-n} d y \\
& =\int_{0}^{\infty}\left|\left\{y \in F_{x}:|x-y|^{\alpha-n}>t\right\}\right| d t \\
& \leq \int_{0}^{|x|^{\alpha-n}}\left|\left\{y \in F_{x}:|x-y|^{\alpha-n}>t\right\}\right| d t \\
& \quad+\int_{|x|^{\alpha-n}}^{\infty}\left|\left\{y \in F_{x}:|x-y|^{\alpha-n}>t\right\}\right| d t \\
& =: I_{1}+I_{2} .
\end{aligned}
$$

It is easy to see that

$$
I_{1} \leq \int_{0}^{|x|^{\alpha-n}}|B(0,|x|)| d t=c|x|^{\alpha}
$$

while using the fact that $n /(n-\alpha)>1$ we find that

$$
\begin{aligned}
I_{2} & \leq \int_{|x|^{\alpha-n}}^{\infty}\left|\left\{y \in F_{x}:|x-y| \leq t^{1 /(\alpha-n)}\right\}\right| d t \\
& \leq c \int_{|x|^{\alpha-n}}^{\infty} t^{n /(\alpha-n)} d t=c_{\alpha, n}|x|^{\alpha} .
\end{aligned}
$$

Finally we conclude that

$$
S_{2}^{\prime}(x) \leq c f\left(\frac{|x|}{2}\right) \leq c H f(x) .
$$

Lower estimate follows immediately by using the fact that $f$ is nonnegative and the obvious estimate $|x-y| \leq|x|$, where $0<|y|<|x|$.

We will also need the following statement.

Lemma 2. Let $r$ be a constant such that $0<r<\infty$. Then the inequality

$$
\begin{array}{r}
\int_{\mathbb{R}^{n}}(H f(x))^{r} u(x) d x \leq C \int_{\mathbb{R}^{n}}(f(x))^{r} u(x) d x, \\
f \in L_{d e c}^{r}\left(u, \mathbb{R}^{n}\right),
\end{array}
$$

holds, if and only if there exists a positive constant $C$ such that, for all $s>0$,

$$
\begin{aligned}
& \int_{|x|>s}\left(\frac{s}{|x|}\right)^{r}|x|^{r(1-n)} u(x) d x \\
& \leq C \int_{|x|<s}|x|^{r(1-n)} u(x) d x .
\end{aligned}
$$

Proof. We will see that inequality (26) is equivalent to the inequality

$$
\int_{0}^{\infty} \widetilde{u}(t)(T \bar{f}(t))^{r} d t \leq C \int_{0}^{\infty} \widetilde{u}(t)(\bar{f}(t))^{r} d t
$$

where $\widetilde{u}(t)=t^{(n-1)(1-r)} \bar{u}(t), \bar{f}(t)=t^{n-1} f(t)$, and $\bar{u}(t)=$ $\int_{S^{n-1}} u(t \bar{x}) d \sigma(\bar{x})$.

Indeed, using polar coordinates in $\mathbb{R}^{n}$ we have

$$
\begin{aligned}
\int_{\mathbb{R}^{n}} & (H f(x))^{r} u(x) d x \\
= & \int_{\mathbb{R}^{n}} u(x)\left(\frac{1}{t^{n}} \int_{|y|<t} f(y) d y\right)^{r} d x \\
= & \int_{0}^{\infty} t^{n-1}\left(\frac{1}{t^{n}} \int_{|y|<t} f(y) d y\right)^{r}\left(\int_{S^{n-1}} u(t \bar{x}) d \sigma \bar{x}\right) d t \\
= & C \int_{0}^{\infty} t^{n-1} t^{-n r} t^{r}\left(\frac{1}{t} \int_{0}^{t} \tau^{n-1} f(\tau) d \tau\right)^{r} \bar{u}(t) d t \\
= & C \int_{0}^{\infty} t^{n-1} t^{r(1-n)} \bar{u}(t)\left(\frac{1}{t} \int_{0}^{t} \bar{f}(\tau) d \tau\right)^{r} d t \\
\leq & C \int_{0}^{\infty} \tilde{u}(t)(\bar{f}(t))^{r} d t \\
= & C \int_{0}^{\infty} t^{(n-1)(1-r)} t^{(n-1) r}(f(t))^{r} d t \\
= & C \int_{\mathbb{R}^{n}}(f(x))^{r} u(x) d x .
\end{aligned}
$$

Conversely taking the test function $f_{r}(x)=$ $\chi_{B(0, r)}(x)|x|^{1-n}, r>0$, in modular inequality (26), one can easily obtain inequality (27). 


\section{The Main Results}

To formulate the main results we need to prove the following proposition.

Proposition 3. Let $u$ be a weight on $\mathbb{R}^{n}$ and $p: \mathbb{R}^{n} \rightarrow \mathbb{R}_{+}$ such that $0<p^{-} \leq p^{+}<\infty$, and assume that $\varphi_{p(\cdot), u(0+)}=0$. The following statements are equivalent:

(a) there exists a positive constant $C$ such that, for any $f \in D R\left(\mathbb{R}^{n}\right)$,

$\int_{\mathbb{R}^{n}}(H f(x))^{p(x)} u(x) d x \leq C \int_{\mathbb{R}^{n}}(f(x))^{p(x)} u(x) d x ;$

(b) for any $r, s>0$,

$$
\int_{|x|>r}\left(\frac{r}{s|x|^{n}}\right)^{p(x)} u(x) d x \leq C \int_{B(0, r)} \frac{|x|^{(1-n) p(x)} u(x)}{s^{p(x)}} d x
$$

(c) $p_{\left.\right|_{\text {supp } u}} \equiv p_{0}$ a.e. and $u \in B_{p_{0}}$.

Proof. We use the arguments of [10]. To show that (a) implies (b) it is enough to test the modular inequality (30) for the function $f_{r, s}(x)=(1 / s) \chi_{B(0, r)}(x)|x|^{1-n}, s, r>0$. Indeed, it can be checked that

$$
H f_{r, s}(x)= \begin{cases}\frac{1}{|x|^{n} s} \int_{|y| \leq|x|}|y|^{1-n} d y, & \text { if }|x| \leq r \\ \frac{1}{|x|^{n} s} \int_{|y| \leq r}|y|^{1-n} d y, & \text { if }|x|>r .\end{cases}
$$

Further, we find that

$$
\begin{aligned}
& \int_{|x|>r} u(x)\left(H f_{r, s}\right)^{p(x)} d x \\
& \leq \int_{\mathbb{R}^{n}} u(x)\left(H f_{r, s}\right)^{p(x)} d x \\
& \leq C \int_{\mathbb{R}^{n}} u(x)\left(\frac{1}{s} \chi_{B(0, r)}(x)|x|^{1-n}\right)^{p(x)} d x .
\end{aligned}
$$

Therefore

$$
\int_{|x|>r} u(x)\left(\frac{r}{s|x|^{n}}\right)^{p(x)} d x \leq C \int_{B(0, r)} \frac{|x|^{(1-n) p(x)} u(x)}{s^{p(x)}} d x .
$$

To obtain (c) from (b) we are going to prove that condition (b) implies that $\varphi_{p(\cdot), u(\delta)}$ is a constant function; namely, $\varphi_{p(\cdot), u(\delta)}=$ $p_{u}^{+}-p_{u}^{-}$for all $\delta>0$. This fact and the hypothesis on $\varphi_{p(\cdot), u(\delta)}$ imply that $\varphi_{p(\cdot), u(\delta)} \equiv 0$, and hence, due to (7),

$$
p_{\left.\right|_{\text {supp } u}} \equiv p_{u}^{+}-p_{u}^{-} \equiv p_{0} \quad \text { a.e. }
$$

Finally (31) means that $u \in B_{p_{0}}$. Let us suppose that $\varphi_{p(\cdot), u}$ is not constant. Then one of the following conditions holds:

(i) there exists $\delta>0$ such that

$$
\alpha=\operatorname{esssup}_{x \in B(0, \delta) \cap \operatorname{supp} u} p(x)<p_{u}^{+}<\infty
$$

and, hence, there exists $\epsilon>0$ such that

$$
|\{|x|>\delta: p(x) \geq \alpha+\epsilon\} \cap \operatorname{supp} u|>0,
$$

or

(ii) there exists $\delta>0$ such that

$$
\beta=\underset{x \in B(0, \delta) \cap \operatorname{esupp} u}{\operatorname{essinf}} p(x)>p_{u}^{-}>0,
$$

and then, for some $\epsilon>0$,

$$
|\{|x|>\delta: p(x) \leq \beta-\epsilon\} \cap \operatorname{supp} u|>0 .
$$

In case (i) we observe that condition (b), for $r=\delta$, implies that

$$
\int_{|x|>\delta}\left(\frac{\delta}{s}\right)^{p(x)} \frac{u(x)}{|x|^{n p(x)}} d x \leq C \int_{B(0, \delta)} \frac{|x|^{(1-n) p(x)} u(x)}{s^{p(x)}} d x .
$$

Then using (36) we obtain, for $s<\min (1, \delta)$,

$$
\begin{aligned}
& \left(\frac{\delta}{s}\right)^{\alpha+\epsilon} \int_{\{|x| \geq \delta: p(x) \geq \alpha+\epsilon\}} \frac{u(x)}{|x|^{n p(x)}} d x \\
& \quad \leq \frac{C}{s^{\alpha}} \int_{B(0, \delta)} u(x)|x|^{(1-n) p(x)} d x,
\end{aligned}
$$

which is clearly a contradiction if we let $s \downarrow 0$. Similarly in case (ii) let us consider the same condition (b), for $r=\delta$, and fix now $s>1$. Taking into account (38) we find that

$$
\begin{aligned}
& \frac{1}{s^{\beta-\epsilon}} \int_{\{|x| \geq \delta: p(x) \leq \beta-\epsilon\}}\left(\frac{\delta}{|x|^{n}}\right)^{p(x)} u(x) d x \\
& \leq \frac{C}{s^{\beta}} \int_{B(0, \delta)}|x|^{(1-n) p(x)} u(x) d x,
\end{aligned}
$$

which is a contradiction if we let $s \uparrow \infty$.

Finally, the fact that condition (c) implies (a) follows from [18, Theorem 1.7].

Theorem 4. Let $u$ be a weight on $(0, \infty)$ and $p: \mathbb{R}_{+} \rightarrow \mathbb{R}_{+}$ such that $0<p^{-} \leq p^{+}<\infty$. Assume that $\psi_{p(\cdot), v\left(0^{+}\right)}=0$. The following facts are equivalent:

(i) there exists a positive constant $C$ such that, for any $f \in$ $D\left(\mathbb{R}_{+}\right)$,

$$
\begin{aligned}
\int_{\mathbb{R}_{+}} & \left(R_{\alpha} f(x)\right)^{p(x)} u(x) d x \\
\leq & C \int_{\mathbb{R}_{+}}(f(x))^{p(x)} u(x) d x ;
\end{aligned}
$$


(ii) condition (13) holds;

(iii) condition (c) of Theorem B is satisfied.

Proof. Proof follows by using Theorem B and Proposition 1(a).

Theorem 5. Let $u$ be a weight on $\mathbb{R}^{n}$ and $p: \mathbb{R}^{n} \rightarrow \mathbb{R}_{+}$such that $0<p^{-} \leq p^{+}<\infty$, and assume that $\varphi_{p(\cdot), u\left(0^{+}\right)}=0$. The following facts are equivalent:

(i) there exists a positive constant $C$ such that, for any $f \in$ $D R\left(\mathbb{R}^{n}\right)$,

$$
\begin{aligned}
& \int_{\mathbb{R}^{n}}\left(I_{\alpha} f(x)\right)^{p(x)} u(x) d x \\
& \leq C \int_{\mathbb{R}^{n}}(f(x))^{p(x)} u(x) d x
\end{aligned}
$$

(ii) condition (31) holds;

(iii) condition (c) of Proposition 3 holds.

Proof. Proof follows by using Propositions 3 and 1(b).

\section{Conflict of Interests}

The authors declare that there is no conflict of interests regarding the publication of this paper.

\section{Acknowledgments}

The authors are grateful to Professor A. Meskhi for drawing their attention to the problem studied in this paper and helpful remarks. The authors are also grateful to the editor and anonymous reviewer for their careful review, valuable comments, and remarks to improve this paper.

\section{References}

[1] D. Cruz-Uribe and A. Fiorenza, Variable Lebesgue Spaces, Birkhäauser, Basel, Switzerland, 2013.

[2] L. Diening, P. Harjulehto, P. Hästö, and M. Ružička, Lebesgue and Sobolev Spaces with Variable Exponents, vol. 2017 of Lecture Notes in Mathematics, Springer, Heidelberg, Germany, 2011.

[3] M. Ružicka, Electrorheological Fluids: Modeling and Mathematical Theory, vol. 1748 of Lecture Notes in Mathematics, Springer, Berlin, Germany, 2000.

[4] L. Diening and S. Samko, "Hardy inequality in variable exponent Lebesgue spaces," Fractional Calculus \& Applied Analysis, vol. 10, no. 1, pp. 1-18, 2007.

[5] D. E. Edmunds, V. Kokilashvili, and A. Meskhi, "On the boundedness and compactness of weighted Hardy operators in spaces $L^{p(x)}$," Georgian Mathematical Journal, vol. 12, no. 1, pp. 27-44, 2005.

[6] D. E. Edmunds, V. Kokilashvili, and A. Meskhi, "Two-weight estimates in $L^{p(.)}$ spaces with applications to Fourier series," Houston Journal of Mathematics, vol. 35, no. 2, pp. 665-689, 2009.

[7] T. S. Kopaliani, "On some structural properties of Banach function spaces and boundedness of certain integral operators,"
Czechoslovak Mathematical Journal, vol. 54(129), no. 3, pp. 791805, 2004.

[8] D. Cruz-Uribe and F. I. Mamedov, "On a general weighted Hardy type inequality in the variable exponent Lebesgue spaces," Revista Matemática Complutense, vol. 25, no. 2, pp. 335367, 2012.

[9] F. I. Mamedov and A. Harman, "On a Hardy type general weighted inequality in spaces," Integral Equations and Operator Theory, vol. 66, no. 4, pp. 565-592, 2010.

[10] S. Boza and J. Soria, "Weighted Hardy modular inequalities in variable $L^{p}$ spaces for decreasing functions," Journal of Mathematical Analysis and Applications, vol. 348, no. 1, pp. 383388, 2008.

[11] S. Boza and J. Soria, "Weighted weak modular and norm inequalities for the Hardy operator in variable $\mathrm{L}^{p}$ spaces of monotone functions," Revista Matemática Complutense, vol. 25, no. 2, pp. 459-474, 2012.

[12] H. Rafeiro and S. Samko, "Hardy type inequality in variable Lebesgue spaces," Annales Academice Scientiarum Fennicae Mathematica, vol. 34, no. 1, pp. 279-289, 2009.

[13] H. Rafeiro and S. Samko, "Corrigendum to Hardy type inequlity in variable Lebesgue spaces," Annales Academiae Scientiarum Fennicae Mathematica, vol. 35, no. 2, pp. 679-680, 2010.

[14] D. E. Edmunds and A. Meskhi, "Potential-type operators in $L^{p(x)}$ spaces," Zeitschrift für Analysis und ihre Anwendungen, vol. 21, no. 3, pp. 681-690, 2002.

[15] U. Ashraf, V. Kokilashvili, and A. Meskhi, "Weight characterization of the trace inequality for the generalized RiemannLiouville transform in $L^{p}(x)$ spaces," Mathematical Inequalities and Applications, vol. 13, no. 1, pp. 63-81, 2010.

[16] V. Kokilashvili, A. Meskhi, and M. Sarwar, "One and two-weight norm estimates for one-sided operator in $L^{p(x)}$ spaces," Eurasian Mathematical Journal, vol. 1, no. 1, pp. 73-110, 2010.

[17] A. Meskhi, Measure of Non-Compactness for Integral Operators in Weighted Lebesgue Spaces, Nova Science Publishers, New York, NY, USA, 2009.

[18] M. A. Arino and B. Muckenhoupt, "Maximal functions on classical Lorentz spaces and Hardy's inequality with weights for nonincreasing functions," Transactions of the American Mathematical Society, vol. 320, no. 2, pp. 727-735, 1990.

[19] E. Sawyer, "Boundedness of classical operators on classical Lorentz spaces," Studia Mathematica, vol. 96, no. 2, pp. 145-158, 1990.

[20] E. T. Sawyer, "Multipliers of Besov and power-weighted $L^{2}$ spaces," Indiana University Mathematics Journal, vol. 33, no. 3, pp. 353-366, 1984.

[21] D. E. Edmunds, V. Kokilashvili, and A. Meskhi, Bounded and Compact Integral Operators, Mathematics and Its Applications, Kluwer Academic, London, UK, 2002.

[22] G. Sinnamon, "Four questions related to Hardy's inequality," in Function Spaces and Applications (Delhi, 1997), pp. 255-266, Narosa, New Delhi, India, 2000.

[23] O. Kováčik and J. Rákosník, "On spaces $L^{p(x)}$ and $W^{k, p(x), "}$ Czechoslovak Mathematical Journal, vol. 41, no. 4, pp. 592-618, 1991.

[24] S. G. Samko, "Convolution type operators in $L^{p(x)}$," Integral Transforms and Special Functions, vol. 7, no. 1-2, pp. 123-144, 1998. 


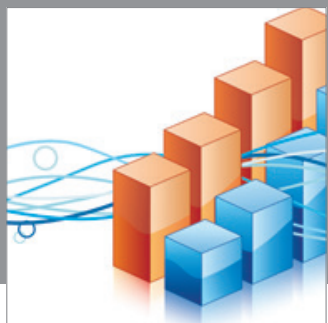

Advances in

Operations Research

mansans

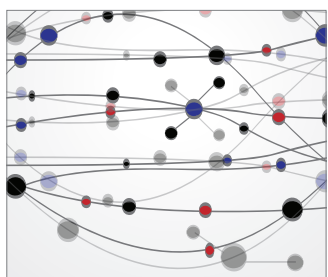

The Scientific World Journal
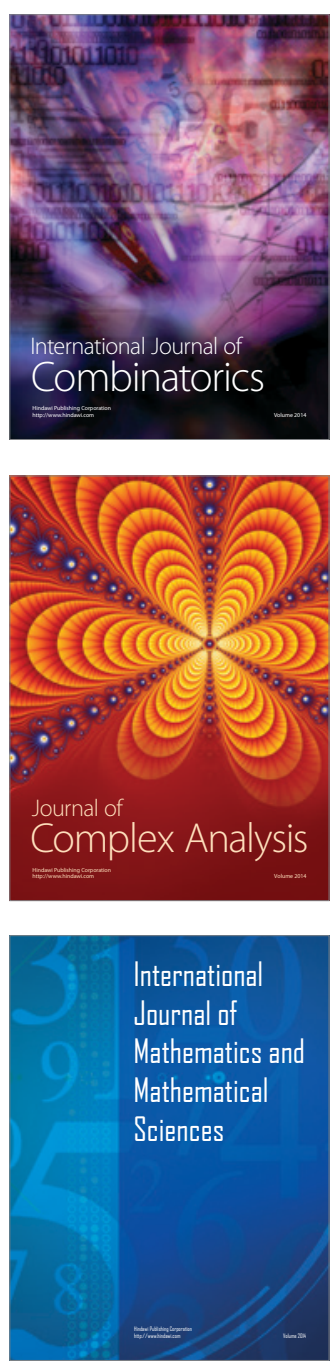
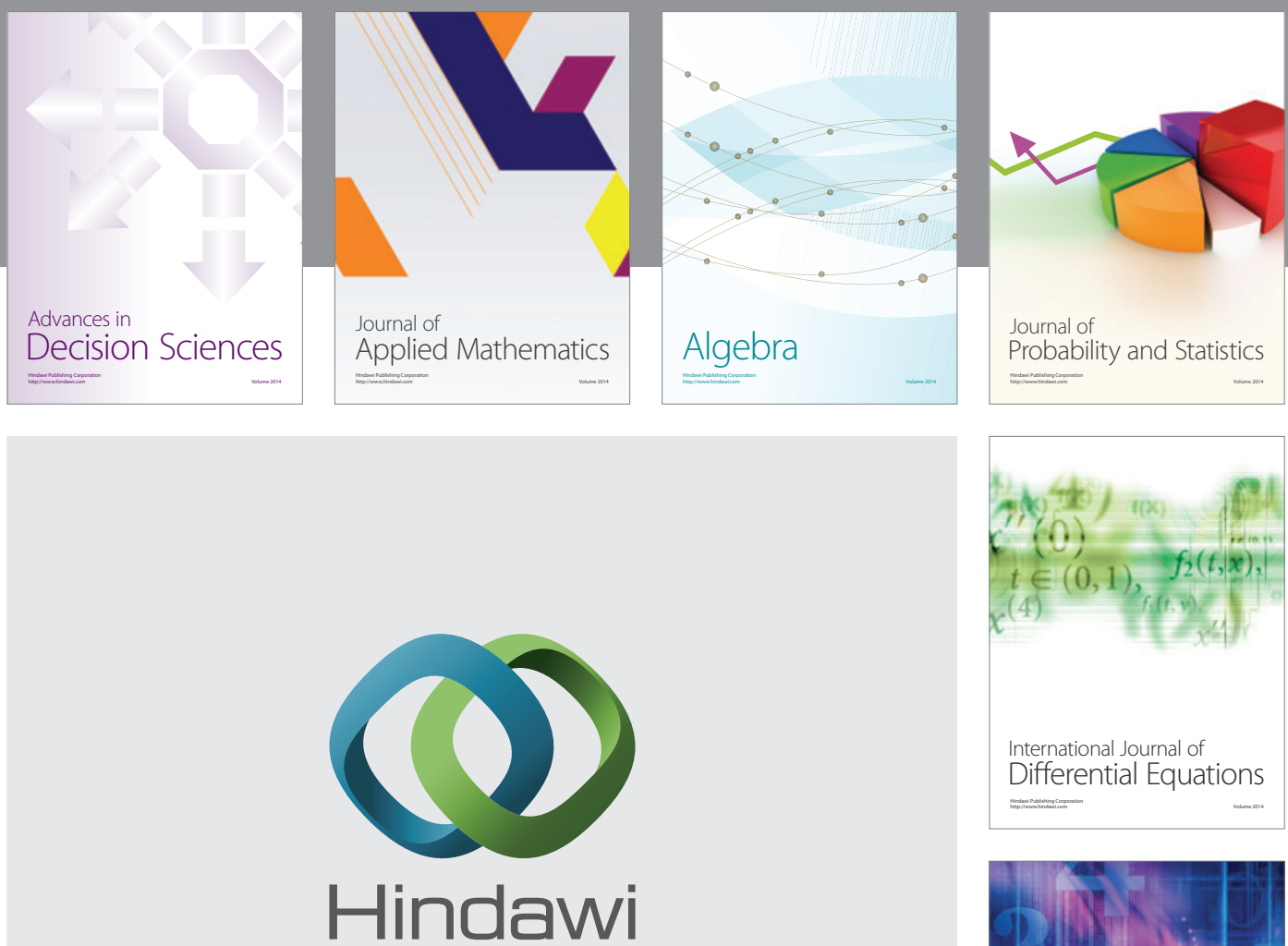

Submit your manuscripts at http://www.hindawi.com
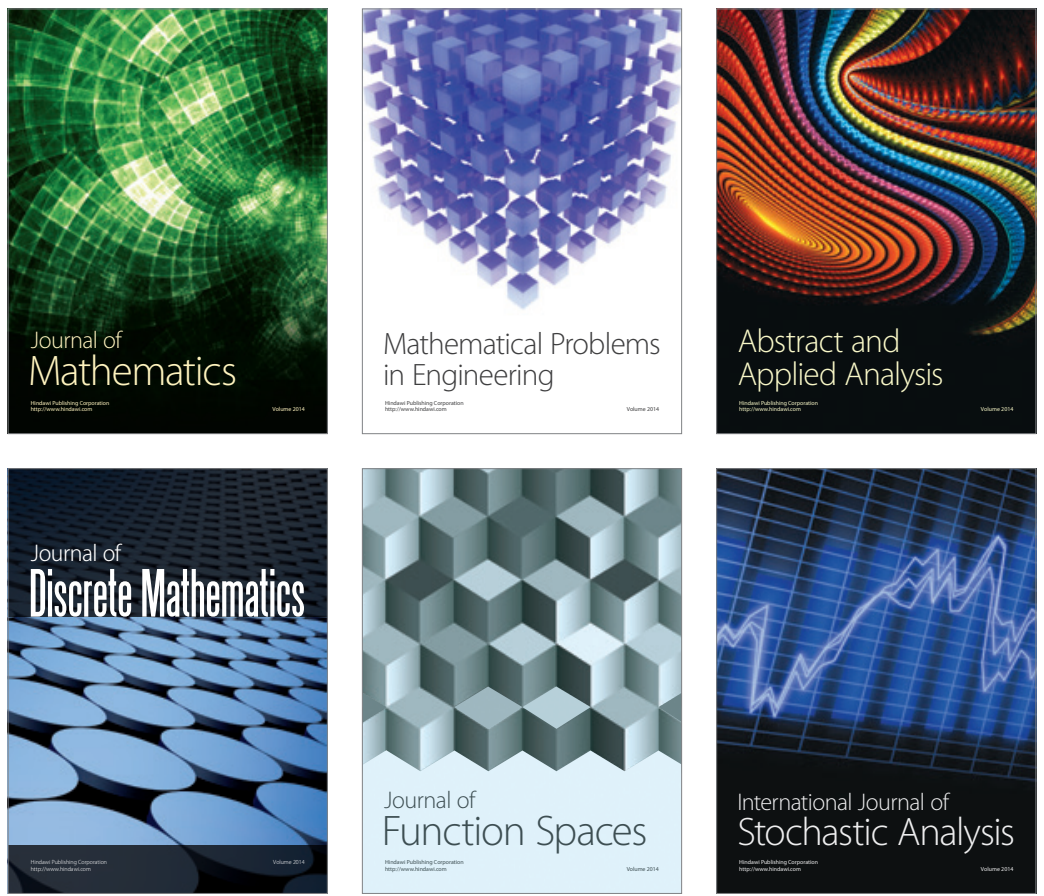

Journal of

Function Spaces

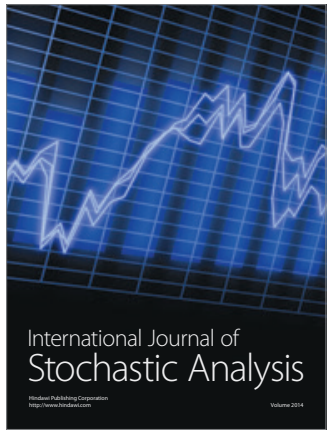

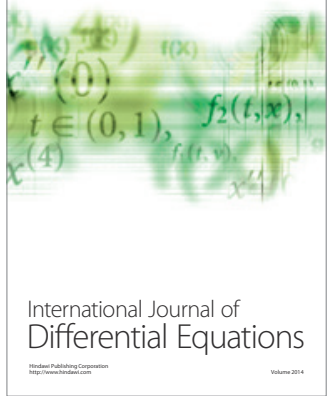
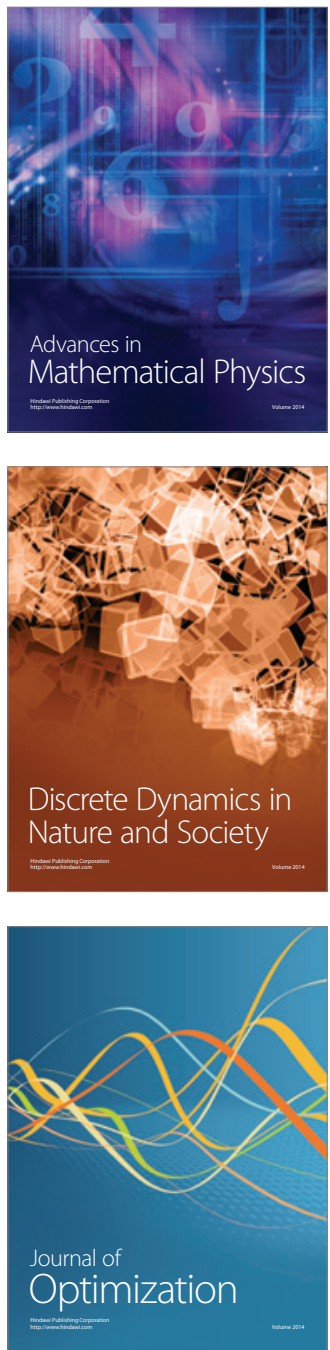